\title{
EFFECT OF THREE DIFFERENT ESTHETIC TREATMENTS ON THE MICROHARDNESS AND SURFACE ROUGHNESS OF (ICON) TREATED TEETH
}

\author{
Ahmed A. Nassar*
}

\section{ABSTRACT}

Objective: The aim of this study was to evaluate the effects of three esthetic treatments on the microhardness and surface roughness of the teeth treated with (Icon) at different storage times Materials and Methods: White spot lesion was created on the labial surfaces of 120 anterior teeth were prepared, treated with Icon resin, divided into 4groups according to the treatment (bleaching, polishing and brushing), with different storage times(one day, one week and one month). Microhardness and surface roughness were measured. Results: There was a decrease in the microhardness and an increase in the surface roughness comparing with the control group specially in the polishing subgroup and after one month of storage. Conclusion: Esthetic treatment affects the microhardness and surface roughness of Icon treated teeth. Polishing has highest effect while, brushing has the lowest. These effects increases with storage.

Key words: ICON, microhardness, surface roughness, bleaching, polishing, brushing

\section{INTRODUCTION}

Filling of anterior teeth has progressed from the normal materials to the treatment by resin infiltration this improved the mechanical properties, optical behavior, brightness, preparation and color, reduce teeth drilling, working time, effort and pain during and after treatment ${ }^{(1)}$. Bleaching, polishing and brushing are three important esthetic treatments used to keep and improve the color of treated teeth, minimize plaque accumulation and gingival irritation but they may have some undesirable effects on their surface properties ${ }^{(2)}$.

The use of polishing brushes and abrasive powered by the polishing motor creates a high friction that can decrease the microhardness and increase the surface roughness of the Icon treated teeth, it has the highest effect among them. It depends on the pressure force, time, abrasive and coolant using ${ }^{(3)}$. The use of bleaching is safer as it has a little effect, it depends on the concentration and the bleaching time, using carbamide peroxide is safer than using hydrogen peroxide ${ }^{(4)}$. Brushing is the safest treatment as a low pressure was exerted using a manual tooth brush and tooth paste which decrease the effect, it depends on the brushing force, time, bristles stiffness and the abrasive particle size in the paste ${ }^{(4)}$. The storage increases these effects because of some changes happened in the surface layer with aging by the effect of storage solution depending on its duration and temperature ${ }^{(5)}$.

\section{MATERIALS AND METHODS}

All the materials compositions are listed according to the manufacturers' profile.

\section{METHODS}

\section{Selection of teeth and grouping of specimens:}

A total 120 freshly sound non carious human anterior teeth, free of cracks and any developmental defects, were used in this study. The teeth were

\footnotetext{
* B.D.S 1997, Mansura University, Dentist, Ministry of Health
} 
TABLE (1): Materials used in this study:

\begin{tabular}{|c|c|c|c|c|}
\hline & Brand name & Composition & Manufacturer \& Website & Batch No. \\
\hline $1-$ & $\begin{array}{l}\text { Icon Kit } \\
\text { Bleaching Kit } \\
\text { (In-Office) }\end{array}$ & $\begin{array}{l}\text { Icon-Etch: Hydrochloric acid, Curing } \\
\text { resins: Pyroginic Silicic acid } \\
\text { Surface-Active (Icon) substance, } \\
\text { Icon-Dry : } 99 \% \text { Ethanol } \\
\text { Icon-Infiltrant: Mety-acrylate base resin } \\
\text { matrix, initiators, additives. } \\
\text { Hydrogen Peroxide } 40 \% \text {, } \\
\text { Water } 20 \% \text {, } \\
\text { Potassium Nitrate, Potassium fluoride. }\end{array}$ & $\begin{array}{l}\text { DMG, } \\
\text { Hamburg, Germany. e-mail: } \\
\text { www. dmg-dental.com }\end{array}$ & 88245 \\
\hline $3-$ & Polishing Kit & $\begin{array}{l}\text { Pumice paste, polishing brushes, polishing } \\
\text { tips, polishing cups, applicators, mandrils }\end{array}$ & $\begin{array}{l}\text { 3M company, USAe.mail:3m. } \\
\text { dental.com }\end{array}$ & 16593 \\
\hline 4- & Brushing Kit & sensodyne tooth paste. & $\begin{array}{c}\text { GSK, USA, } \\
\text { e-mail: gsk.dent.com. }\end{array}$ & 77239 \\
\hline $5-$ & Demineralizing gel. & $37 \%$ Phosphoric acid. & $\begin{array}{c}\text { 3M company USA e.mail:3m. } \\
\text { dental.com }\end{array}$ & 30075 \\
\hline
\end{tabular}

divided into two main groups (60 teeth each) according to the testing technique: group $(\mathrm{H})$ : used for assessment of microhardness and group (R): used for assessment of surface roughness. Each group was subdivided into four equal subgroups (15 teeth each) according to the esthetic treatment as follows: subgroup (A): control, (treated with Icon but no esthetic treatments), the other three subgroups were treated with Icon, then treated as the following: subgroup (B): treated with the bleaching kit, Subgroup (C): treated with the polishing kit, and Subgroup (D): treated with brushing kit. Each subgroup was further subdivided into three equal divisions (5 teeth each) according to the storage times as follows: (S1) one day, (S2) one week and (S3) one month.

\section{1-Preparation of the specimens;}

The crowns of 120 human anterior teeth were separated and embedded horizontally in its middle of a circle plastic mould of internal diameter $10 \mathrm{~mm}$ and $20 \mathrm{~mm}$ in height leaving $2 \mathrm{~mm}$ from the labial surface projecting.

\section{B- Enamel white spot creation:}

Labial surfaces of specimens were treated with $37 \%$ phosphoric acid for $1 \mathrm{~min}$.

\section{C- Icon application:}

Icon was applied as: Icon-Etch was applied, allowed to sit for $2 \mathrm{~min}$, rinsed with water air spray for $30 \mathrm{sec}$, then, air dried with gentle pressure for $5 \mathrm{sec}$. Icon-Dry was applied, left for $30 \mathrm{sec}$. air dried for $5 \mathrm{sec}$. Icon-Infiltrant was applied, allowed to sit for $3 \mathrm{~min}$, light-cured for $40 \mathrm{sec}$. application was repeated ${ }^{(6)}$.

\section{D- Esthetic treatments:}

1- Control subgroup: specimens immersed in a daily changed artificial saliva.

2- Bleaching subgroup: application was done on 2 sessions 20 minutes each then measurements were made. After one day, one week and one month, specimens were subjected to the bleaching procedure then, measurements were done. 
3- Polishing subgroup: specimens were put in a tooth-polishing simulator apparatus where a polishing brush was rotated by a conventional latched type contra angle hand piece fixed upright stand and the brush parallel horizontally to a plate which has an acrylic base with metal ring for holding the specimen with a polishing paste and fixed load at $150 \mathrm{~g}$ for $4-5 \mathrm{sec}$, then, stored in an incubator till tested after one day, one week and one month.

4- Brushing Subgroup: specimens were put in a tooth-brush simulator apparatus where a battery powered electric brush mounted to a fixed upright stand and the brush was placed parallel horizontally to a plate which has an acrylic base with metal ring for holding the specimen with a $250 \mathrm{~g}$ load for $2 \mathrm{~min}$ per day using a slurry of sensodyne toothpaste, stored in the incubator till measuring after one day, one week and one month.

\section{F- Microhardness assessment:}

At a minimum of 3 separated locations with equal distances on the middle third of the labial surface of each specimen, indentations were made using a Vickers diamond indenter with a 2-N load and for a 15-S dwell time. Recorded values were averaged to produce the mean hardness value for each specimen ${ }^{(7)}$.

\section{G- Surface roughness assessment:}

Enamel Surface roughness of each specimen was

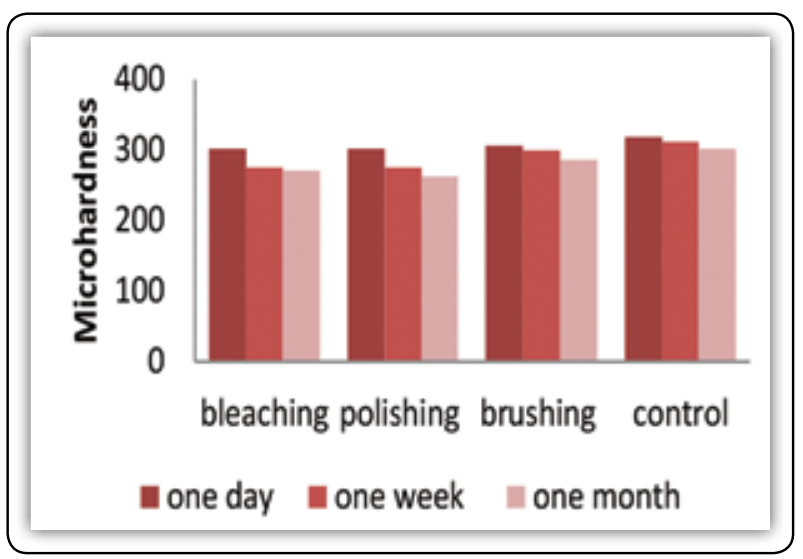

Fig. (1) Histogram of the of hardness as a function of time determined using non- contact type profilometer s, with magnification of $\mathrm{x} 20$. Recommends cutoffs $0,8 \mathrm{~mm}^{(8)}$.

\section{II-RESULTS}

\section{A- Microhardness:}

The control group recorded the highest microhardness mean value. While, all the esthetic treatments recorded the lowest microhardness mean values starting with brushing treated group, then bleaching treated group, finally the polishing treated group which recorded the lowest mean value of the microhardness. The one day stored subgroups recorded the highest microhardness mean values followed by one week stored subgroups while, one month stored subgroups recorded the lowest microhardness mean value.

\section{B- Surface roughness:}

The control group where Icon was applied with no esthetic treatments recorded the lowest roughness mean value than any treatment group. All the esthetic treatments caused an increase in the surface roughness mean values starting with brushing treated group, then, the bleaching treated group, finally, the polishing treated group which recorded the highest mean value of the surface roughness. The one month stored sub- groups recorded the highest roughness mean values followed by one week stored subgroups while one day stored subgroups recorded the lowest roughness mean value.

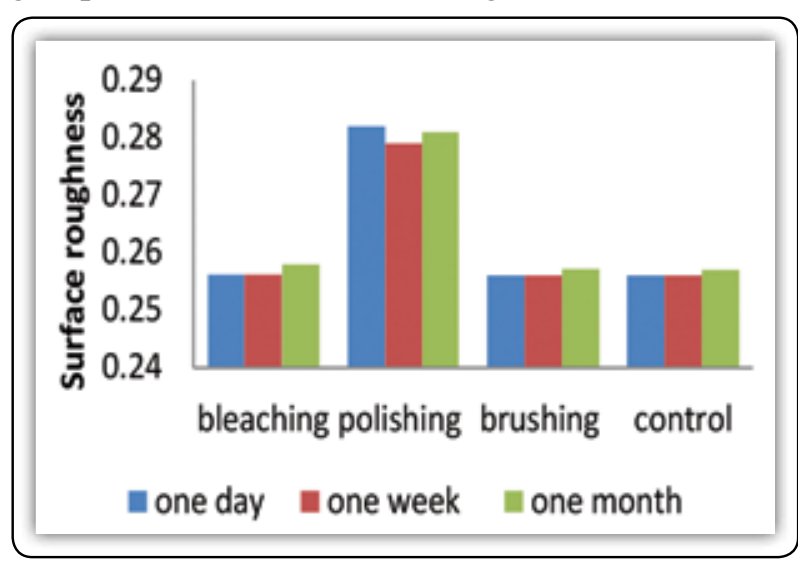

Fig. (2) Histogram of the of roughness as a function of time . 


\section{DISCUSSION}

The control group recorded the highest microhardness and the lowest surface roughness mean value. This may be due to: The Icon prevents further progression of enamel lesions by occluding the microporosities ${ }^{\left({ }^{9}\right)}$. It penetrates into the lesion body driven by capillary forces, improving the surface of the treated teeth ${ }^{(10)}$. However, the brushing treated group recorded a mean value lower than control group but higher than the other groups. This may be due to: the brushing force is a small frictional force affects the wear behavior of the surface of infiltrated teeth ${ }^{(11)}$. Also, polishing treated group recorded the lowest mean value .This may be due to: polishing causes the surface molecules of infiltrated teeth to be loose bonded and the polishing brush causes some changes on the tooth surface . This energy is related to the speed, pressure, abrasive size and coolant ${ }^{(12)}$ Moreover, bleaching was made by the action of certain chemicals on the surface of infiltrated teeth. The bleaching agents remove stains by oxidizing the organic substances within the tooth structure which proved to release of free radicals which moves freely between the surface layers causing bonding weakening ${ }^{(13)}$.

\section{CONCLUSION}

Within the limitations of this in vitro study, the following conclusions may be drawn:

1. Icon improves the surface of enamel of white spot lesions.

2. Esthetic treatments affect the surface properties of the Icon treated teeth.

3. Polishing treatment has the highest effect while brushing has the lowest effect.

4. The storage time has an adverse effect on the surface properties of the Icon treated teeth.

\section{REFERENCES}

1. Meyer-Lueckel H, Paris S: Progression of artificial enamel caries lesions after infiltration with light-curing resins. Caries Res 2003; 42:117-24.

2. Yap A, Sau C, Lye K. Effects of finishing and polishing time on surface characteristics of tooth-coloured restoratives. J Oral Rehab. 1998; 25:456-61.

3. Neme A, Wagner W, Pink FE, Frazier KB. The effect of prophylactic polishing pastes and tooth brushing on the surface roughness of resin composite materials in vitro. Oper Dent 2003; 28:808-15.

4. Paris S, Meyer-Luckel H, KielbassaAM. Resin Infiltration of natural caries lesion. J Dent Res 2007; 86:662-6.

5. Liu Y, Deng H, Tang L, Zhang Z. Effect of resin infiltration on micro-hardness of artificial caries lesions. J Kor Acad Pediatr Dent J 2015; 50:737-41.

6. Mohammed F and Qasim A. Assessment of white spot lesions treated with Icon and fluoride gel. Enh Res in Sci Tech and Eng $\mathbf{J} 2014 ; 3: 1-6$.

7. Zamorano P, Valenzuela A, Vial P, Vidal M. Microhardness of artificial white spot lesions infiltrated with low viscosity resin before and after thermocycling. Oper Dent J $2015 ; 31: 371-8$

8. Tostes M, Santos e, Camargo S. Effect of resin infiltration on the nanomech-anical properties of demineralized bovine enamel. Indian Dent J 2014; 5:116-22.

9. Okte Z, Villalta P, Garcia F, Lu H, Powers JM. Surface hardness of resin composites after staining and bleaching. Oper Dent J 2006; 31:623-8.

10. Christian H, Sebastian D, Klaus B, Edgar B, Elke K, et al Effect of bleaching on subsurface micro-hardness of composite and a polyacid modified composite. Dent Mater J 2006; 910:1-6.

11. Patel B, Chhabra N, Jain D. Effect of different polishing systems on the surface roughness of nano-hybrid composites. Conserv Dent J 2016; 19:37-41.

12. Arends J, Schuthof J, Jongebloed W. Microhardness indentations on artificial white spot lesions. Caries Res 1979; 13:290-7.

13. Meyer-Luckel H and Paris S. Improved resin infiltration of natural caries lesions. J Dent Res 2008; 87:1112-6. 